\title{
Factores de Riesgo Cardiovasculares en Trabajadores de una Empresa Productora de Grasas y Aceites Vegetales
}

\section{Cardiovascular Risk Factors in Workers of a Company Producer of Fats and Vegetable Oils}

\author{
Mendinueta Martínez $\mathrm{M}^{1}$, Herazo Beltrán $\mathrm{Y}^{2}$ \\ ${ }^{1}$ Maestría en Auditoria y Sistemas de Calidad en Servicios de Salud - Universidad Simón Bolívar, ${ }^{2}$ Programa de Fisioterapia - Universidad Simón Bolívar
}

Resumen - El riesgo cardiovascular se define como la probabilidad que tiene un sujeto de presentar una enfermedad cardiovascular. Objetivo: Determinar los factores de riesgo cardiovascular en trabajadores de una empresa productora de grasas y aceites vegetales. Materiales y Métodos: Estudio de corte transversal en 113 trabajadores. Se indagó sobre variables sociodemográficas, consumo de tabaco y de alcohol, número de días y porciones de frutas y verduras a la semana, antecedentes personales de enfermedad cardiovascular y niveles de actividad física. Además, se midió la talla y el peso para definir el índice de masa corporal. Para el análisis de los datos se utilizó la base de datos SPSS versión 22, se determinó la correlación entre el promedio del índice de masa corporal y el consumo energético en MET a través del coeficiente de correlación lineal.

Resultados: El 44,2\% presentaron sobrepeso, un $64,6 \%$ de los empleados consumen alcohol y el $56,6 \%$ no cumplen las recomendaciones de actividad física en el tiempo libre. El promedio de días consumo de frutas es de 2,8 días y el número de porciones de 1,6 frutas. Se encontró una correlación negativa y significativa $(-0,18$; $p=0,04)$ entre el consumo energético durante el dominio laboral y el índice de masa corporal.

Conclusión: La detección precoz de los factores de riesgo cardiovasculares es relevante porque permite la implementación de programas de promoción de la salud y prevención en el ámbito laboral.

Palabras Claves: Evaluación, Conocimiento, Guía de Práctica Clínica (GPC), Barreras, personal de salud, Trastornos Hipertensivos (Fuente: DeCS).

\footnotetext{
Abstract - Cardiovascular risk is defined as the probability that a subject of developing cardiovascular disease in adulthood.

Objective: To determine the cardiovascular risk factors in workers of a producer of fats and vegetable oils.

Materials and methods: study of cross-section in 113 workers. Asked about socio-demographic variables, tobacco and alcohol consumption, number of days and lots of fruits and vegetables a week, personal
}

Correspondencia: Martha Mendinueta Martínez. Programa de Fisioterapia, Facultad de Ciencias de la Salud. Universidad Simón Bolívar. Cr 59 \# 59 - 65. Barranquilla, Atlántico, Colombia.mmendinueta@unisimonbilivar.edu.co.

Citar: Mendinueta Martínez M, Herazo Beltrán Y. Factores de Riesgo Cardiovasculares en Trabajadores de una Empresa Productora de Grasas y Aceites Vegetales. Cienc e Innovación en Salud [Internet]. 2015 Jan 1;3(1):23-26. Available from: http://publicaciones.unisimonbolivar.edu.co:82/rdigital/ojs/index.php/innovacionsalud/article /view/235/232

Parte de este trabajo se encuentran soportado en la investigación titulada « Factores de Riesgo Cardiovasculares en Trabajadores de una Empresa Productora de Grasas y Aceites Vegetales» tesis para obtener el título de Magíster en en Auditoria y Sistemas de Calidad en Servicios de Salud de la Universidad Simón Bolivar (Colombia).

Recibido: Oct. 12 de 2014 / Modificado: Nov. 11 de 2014 / Aceptado: Nov. 21 de 2014 cardiovascular disease history and levels of physical activity. In addition, measured height and weight to define the body mass index. The analysis of the data was SPSS database version 22, determined the correlation between body mass index and average energy consumption in MET through the linear correlation coefficient.

Results: $44,2 \%$ were overweight, a 64.6 percent of employees use alcohol and $56,6 \%$ do not meet recommendations for physical activity in their free time. The average number of days consumption of fruit is 2.8 days and the number of servings of fruit 1.6. A negative and significant correlation $(-0,18 ; p=0,04)$ between the energy consumption during the work domain and body mass index. Conclusion: Early detection of cardiovascular risk factors is relevant because it allows the implementation of programs for the promotion of health and prevention in the workplace.

Keywords: Assessment, Knowledge, Clinical Practice Guideline (CPG), barriers, personal health, Hypertensive disorders. (Source: MeSH, NLM).

\section{INTRODUCCIÓN}

El riesgo cardiovascular se define como la probabilidad que tiene un sujeto de presentar una enfermedad cardiovascular $(1,2)$. Las enfermedades cardiovasculares hacen parte de las enfermedades crónicas no transmisibles incluyendo patologías cerebrovasculares y cardiacas; estás producen 17.3 millones de muertes al año en todo el planeta (3) y son responsables de 32 millones de eventos tanto coronarios como accidentes cerebro-vasculares de los cuales, entre el 40$70 \%$, son fatales en países desarrollados, así como en países en vía de desarrollo (4). Ubicándola como la primera causa de muerte a nivel mundial lo que genera un grave problema de salud pública $(3,5,6)$, por la alta incidencia en la morbi-mortalidad de las personas (5).

Existen múltiples factores asociados con esta patología los cuales pueden ser de tipo modificable como el consumo de alcohol, hiperlipidemia, uso de anticonceptivos, tabaquismo, sedentarismo, hiperglicemia, obesidad y malos hábitos alimenticios; y no modificables como el sexo, la raza, edad y los antecedentes familiares de enfermedad cardiovascular (7). 
En países como España las enfermedades cardiovasculares representan el $31 \%$ de todas las muertes (8). En áfrica subsahariana estas enfermedades ocasionaron el $28 \%$ de la morbilidad y el $35 \%$ de la mortalidad, se estima que para el 2020 se incrementen de un $60 \%$ a un $65 \%$ (9). Estados Unidos no es ajeno a esta patología, para el año 2007 representaron 261 muertes por cada 100.000 hombres y 273 muertes por cada 100.000 mujeres representando el 33\% de la mortalidad total (10). En las Américas son la causa de 1.9 millones de muertes al año lo que constituye grandes pérdidas económicas (5), en los últimos 20 años la prevalencia de estas enfermedades ha aumentado de manera significativa en México, según un estudio realizado el $70 \%$ de la población se encontraba en sobrepeso y obesidad, el $26,5 \%$ colesterol y el $13,5 \%$ diabetes (11). En Colombia según el Instituto Nacional de Salud, para el año 2008 ocasionaron el 30,1\% de todas las muertes y es registrada como la primera causa de morbi-mortalidad (12). Asimismo, en Barranquilla para el año 2010 ocasionó la muerte del $76 \%$ y $44 \%$ por 100000 habitantes (13).

Teniendo en cuenta la problemática que generan los factores de riesgo cardiovascular y con base en los lineamientos del Plan Decenal de Salud Pública 20122022, es importante generar estrategias enfatizadas al mejoramiento de las condiciones de salud de toda la población trabajadora, a través del autocuidado y la promoción de entornos laborales saludables en el sector empresarial (14), es por ello que el presente estudio tiene como objetivo determinar los factores de riesgo cardiovascular en trabajadores de una empresa productora de grasas y aceites vegetales en la ciudad de Barranquilla cuyos resultados permitan la creación de estrategias encaminadas a la sensibilización y el fomento del autocuidado.

\section{MATERIALES Y MÉTODOS}

Se realizó un estudio de corte transversal en 113 trabajadores de una empresa cuya actividad económica es la producción de alimentos. Se estudió toda la población contratada por la compañía durante el periodo de la medición. La información se obtuvo mediante una encuesta que indagó sobre variables socio-demográficas como edad, género y área de trabajo; igualmente, sobre consumo de tabaco y de alcohol, el número de días y porciones de frutas $\mathrm{y}$ verduras a la semana, antecedentes personales de enfermedad cardiovascular y el autoreporte de hipertensión, dislipidemia y diabetes; se midió la talla y el peso para definir el índice de masa corporal.
Se evaluaron los niveles de actividad física en el trabajo, tiempo libre y transporte a través del Cuestionario Internacional de Actividad Física, formato largo, el cual permitió categorizar a los sujetos en activos e inactivos según si cumplían o no las recomendaciones de 150 minutos de intensidad moderada o 75 minutos de intensidad vigorosa durante la semana.

Para el análisis de los datos se utilizó la base de datos SPSS versión 22, las variables categóricas se presentan en frecuencias absolutas y relativas. Para las variables continuas se emplea una medida de tendencia central, la media y la desviación estándar. Se determinó la correlación entre el promedio del índice de masa corporal y el consumo energético en MET a través del coeficiente de correlación lineal.

\section{REsULTADOS}

Las características generales de los sujetos estudiados se muestran en la Tabla 1, resaltando que el $94,7 \%$ son hombres, la mayoría son operarios ( $88,5 \%$ ); y aunque el $49,6 \%$ son delgados, se observa que un $44,2 \%$ presentan sobrepeso, considerado un factor de riesgo cardiovascular.

Tabla 1. Características generales de los sujetos de estudio

\begin{tabular}{lccc}
\hline \hline Variable & Nivel & Frec. & $\%$ \\
\hline \multirow{2}{*}{ Genero } & Masculino & 107 & $94,7 \%$ \\
& Femenino & 6 & $5,3 \%$ \\
\hline \multirow{2}{*}{ Área de trabajo } & Administrativa & 13 & 11,5 \\
& Operario & 100 & 88,5 \\
\hline \multirow{2}{*}{ Índice de Masa corporal } & Delgado & 2 & $1,8 \%$ \\
& Normal & 56 & $49,6 \%$ \\
& Sobrepeso & 50 & $44,2 \%$ \\
& Obeso & 5 & $4,4 \%$ \\
\hline \hline Total & & 113 & $100,0 \%$ \\
\hline \hline Fuente: Datos del Estudio & & &
\end{tabular}

En relación con el índice de masa corporal, la Tabla 2 muestra una media de 24,8 y llama la atención que el valor que con mayor frecuencia se repitió fue el de 27,7 $\mathrm{kg} / \mathrm{m} 2$.

Tabla 2. Medidas de tendencia para la edad y el Índice de Masa Corporal

\begin{tabular}{ccc}
\hline \hline & Edad & Índice de masa corporal \\
\hline Media & 32,1 & 24,8 \\
Desviación Estándar & 10 & 3,2 \\
Moda & 29 & 27,7 \\
Rango & $19-63$ & $17-35$ \\
\hline \hline Fuente: Datos del Estudio & &
\end{tabular}

Se observa en la Tabla 3 que el $64,6 \%$ de los empleados consumen alcohol; en lo que respecta a la actividad física, menos trabajadores fueron categorizados como activos durante su tiempo libre $(56,6 \%)$ al comparar con las frecuencias obtenidas en los 
niveles de actividad física en el ámbito laboral o como medio de transporte $(79,6 \%$ y $75,2 \%$, respectivamente). Al indagar sobre si recibían tratamiento para dislipidemia, el $12,4 \%$ de ellos respondieron afirmativamente.

Tabla 3. Factores de riesgo cardiovasculares

\begin{tabular}{|c|c|c|c|}
\hline Variable & Nivel & Frec. & $\%$ \\
\hline \multirow{2}{*}{ Consumo de Tabaco } & $\mathrm{Si}$ & 4 & $3,5 \%$ \\
\hline & No & 109 & $96,5 \%$ \\
\hline \multirow{2}{*}{ Consumo de Alcohol } & $\mathrm{Si}$ & 73 & $64,6 \%$ \\
\hline & No & 40 & $35,4 \%$ \\
\hline \multirow{2}{*}{$\begin{array}{l}\text { Nivel de Actividad Física } \\
\text { en el ámbito laboral }\end{array}$} & Activos & 90 & $79,6 \%$ \\
\hline & Inactivos & 23 & $20,4 \%$ \\
\hline \multirow{2}{*}{$\begin{array}{l}\text { Nivel de Actividad Física } \\
\text { en el ámbito transporte }\end{array}$} & Activos & 85 & $75,2 \%$ \\
\hline & Inactivos & 28 & $24,8 \%$ \\
\hline \multirow{2}{*}{$\begin{array}{l}\text { Nivel de Actividad Física } \\
\text { en el tiempo Libre }\end{array}$} & Activos & 49 & $43,4 \%$ \\
\hline & Inactivos & 64 & $56,6 \%$ \\
\hline \multirow{2}{*}{$\begin{array}{l}\text { Recibe tratamiento } \\
\text { médico para dislipidemia }\end{array}$} & $\mathrm{Si}$ & 14 & $12,4 \%$ \\
\hline & No & 99 & $87,6 \%$ \\
\hline \multirow{2}{*}{$\begin{array}{l}\text { Recibe tratamiento } \\
\text { médico para la } \\
\text { hipertensión arterial }\end{array}$} & $\mathrm{Si}$ & 8 & $7,1 \%$ \\
\hline & No & 105 & $92,9 \%$ \\
\hline \multirow{2}{*}{$\begin{array}{l}\text { Recibe tratamiento } \\
\text { médico para la } \\
\text { hiperglucemia }\end{array}$} & $\mathrm{Si}$ & 5 & $4,4 \%$ \\
\hline & No & 108 & $95,6 \%$ \\
\hline \multirow{2}{*}{$\begin{array}{l}\text { Antecedentes familiares } \\
\text { de enfermedades } \\
\text { crónicas }\end{array}$} & $\mathrm{Si}$ & 44 & $38,9 \%$ \\
\hline & No & 69 & $61,1 \%$ \\
\hline
\end{tabular}

En relación con el consumo de frutas y verduras, la Tabla 4 muestra que el promedio de días consumo de frutas es bajo, de 2,8 días y el número de porciones muy bajos también, 1,6 frutas. Igual comportamiento se observó con el número de porciones de verduras, sólo 1,9 frutas.

Tabla 4. Medidas de tendencia para el consumo de frutas y verduras

\begin{tabular}{lcccc}
\hline & Media & $\begin{array}{c}\text { Desviación } \\
\text { Estándar }\end{array}$ & Moda & Rango \\
\hline $\begin{array}{l}\text { Días de consumo de } \\
\text { frutas }\end{array}$ & 2,8 & 2 & 2 & $0-7$ \\
$\begin{array}{l}\text { Número de porciones } \\
\text { de frutas }\end{array}$ & 1,6 & 1,5 & 1 & $0-7$ \\
$\begin{array}{l}\text { Días de consumo de } \\
\text { verduras }\end{array}$ & 5,6 & 2 & 7 & $0-7$ \\
$\begin{array}{l}\text { Número de porciones } \\
\text { de verduras }\end{array}$ & 1,9 & 1,2 & 1 & $0-5$ \\
\hline \hline Fuente: Datos del Estudio & & & &
\end{tabular}

Al correlacionar el consumo energético durante los tres dominios o ámbitos de la actividad física con el promedio de índice de masa corporal, se observa en la Tabla 5, una relación negativa, es decir a mayor realización de actividad física en el trabajo o el uso de la bicicleta y el ir caminando a la empresa, menor es el índice de masa corporal.

Tabla 5. Correlación lineal entre el índice de masa corporal y el consumo energético (MET) durante actividades físicas

\begin{tabular}{lcc}
\hline \hline & Correlación de Pearson & Valor de $\mathbf{p}$ \\
\hline $\begin{array}{l}\text { IMC-MET en el ámbito } \\
\text { laboral }\end{array}$ & $-0,18$ & 0,05 \\
$\begin{array}{l}\text { IMC-MET en el ámbito } \\
\text { transporte }\end{array}$ & $-0,13$ & 0,15 \\
$\begin{array}{l}\text { IMC-MET en el ámbito } \\
\text { tiempo libre }\end{array}$ & 0,03 & 0,75 \\
\hline \hline
\end{tabular}

\section{DISCUSIÓN}

El presente estudio muestra una alta frecuencia de trabajadores con sobrepeso al compararla con la prevalencia general en Colombia que es del 34,6\% y similar a lo reportado por Martínez (40,1\%) (1) y Alonso (49\%) (15); este hallazgo unido a los demás factores de riesgo encontrados, específicamente un alto consumo de alcohol y patrones alimentarios inadecuados por el bajo consumo de frutas y verduras, colocan a la empresa en la necesidad de implementar medidas de promoción de la salud y prevención de la enfermedad.

En relación con el consumo de tabaco, el reporte fue muy bajo al relacionarlo con otros estudios, donde un $35 \%$ de los trabajadores encuestados eran fumadores activos (15).

Como sucede en otros estudios la inactividad física fue mayor durante el tiempo libre, entendiendo que la mayoría de los participantes del estudio son operarios existe una mayor probabilidad de ser activo físicamente durante el trabajo o el transporte (16); la evaluación de la práctica de actividad física está mediada por el dominio, considerado como las oportunidades de la vida cotidiana donde las personas puedan realizarla, por ello, el tipo de ocupación de la persona determina si se es activo en el ámbito laboral o en el tiempo libre.

Los participantes del presente estudio reportan mayores niveles de actividad por las actividades laborales que realizan relacionadas con el área de trabajo operario, que implican levantamiento de carga, manipulación de equipos, mantenimiento preventivo y correctivo de maquinaria entre otras actividades.

Este tipo de estudio es relevante porque evidencia el compromiso de la empresa en la detección precoz de los factores de riesgo cardiovasculares y por la posibilidad que los trabajadores acudan al sistema de salud toda vez que las personas en edad laboral solicitan consulta médica según las patologías percibidas (17).

Los programas de promoción de la salud en el sitio de trabajo pueden modificar factores de riesgo como inactividad física, tabaquismo, hipertensión, dislipidemia, mala alimentación, hiperglucemia y estrés psicológico elevado; y aunque los empleadores 
consideren que la tarea de mejorar la salud de sus empleados le corresponde a las instituciones de salud, debido a las jornadas de trabajo las personas no pueden asistir a los servicios de atención, el escenario laboral entonces se convertiría en una opción para fomentar estilos de vida saludable $(18,19)$.

El diseño de corte transversal en una limitación de esta investigación porque no permite establecer inferencias causales entre las variables estudiadas, aunque si la postulación de hipotesis causales que permitan plantear futuras investigaciones.

\section{REFERENCIAS BIBLIOGRÁFICAS}

1. Martínez AB, Arbués ER. Prevalencia de los factores de riesgo cardiovascular en trabajadores de los sectores laborales secundario y terciario. Enferm. glob. 2012; 11 (28): 31-40.

2. Vega AJ, Guimará MM, Vega AL. Riesgo cardiovascular, una herramienta útil para la prevención de las enfermedades cardiovasculares. Rev Cubana Med Gen Integr. 2011; 27(1): 91-97.

3. González D, Barguil Z, García Al. Presencia de factores de riesgo cardiovascular en trasportadores de carga pesada, según criterios ATPIII, para síndrome metabólico. Ciencia \& Salud. 2014; 3(9):2327.

4. Lares M, Velazco $\mathrm{Y}$, Brito S, Hernández P, Mata C. Evaluación del estado nutricional en la detección de factores de riesgo cardiovascular en una población adulta. Revista Latinoamericana de Hipertensión. 2011; 6 (1): 8-13.

5. Prieto BB, Amaya RM. Estrategia educativa en salud cardiovascular para trabajadores de una institución educativa. Salud Uninorte. 2014; 30(1):44-51.

6. Vallejo M. Acerca de la necesidad de estudios sobre la incidencia de factores de riesgo cardiovascular en México: ¿Una realidad? Arch. Cardiol. Méx. 2014; 84 (2): 69-70.

7. Magalhaes J, Bento L, Brasil C, Fatores de risco para doencas cardiovasculares em profissionais de enfermagem: estratégias de promocao da saúde. Revista Brasileira de enfermagen reben. 2014. 67(3):394-400.

8. Ramírez de Arellano A, Coca A, De la Figuera M, Rubio TC, Rubio RD, Gracia A, et al. Economic evaluation of Cardio inCode ${ }^{\circledR}$, a clinical-genetic function for coronary heart disease risk assessment. Appl Health Econ Health Policy. 2013; 11(5):531-42.

9. Amuyunzu M. Need for a multi-factorial, multisectorial and multi-disciplinary approach to NCD prevention and control in Africa. Glob Health
Promot. 2010;17 (2 Suppl):31-32.

10. Morales LS, Flores YN, Leng $M$, Sportiche N, Gallegos CK, Salmerón J. Risk factors for cardiovascular disease among Mexican-American adults in the United States and Mexico: a comparative study. Salud pública Méx. 2014; 56 (2): 197-205.

11. Cerecero P, Hernandez PB, Denova $E$, Valdés $R$, Vázquez G, Camarillo $E$, et al. Association between serum uric acid levels and cardiovascular risk among university workers from the State of Mexico: a nested case-control study. BMC Public Health. 2013; 13 (415): 1-9.

12. Machado AJ, Machado DM. Prevalencia de factores de riesgo cardiovascular en pacientes con dislipidemia afiliados al sistema de salud en Colombia. Rev Peru Med Exp Salud Pública. 2013; 30 (2): 205-211.

13. Lizarazu DI, Rossi TC, Iglesias AJ, Villanueva TD. Prevalencia de factores de riesgo cardiovascular y evaluación del riesgo cardiovascular global en trabajadores de la Universidad Libre seccional Barranquilla, 2010 (Colombia). Salud Uninorte. 2013; 29 (1): 52-63.

14. Ministerio de Salud y Protección Social. Plan Decenal de Salud Pública 2012-2021: La salud en Colombia la construyes tú. Bogotá; 2013.

15. Alonso DJ, Calleja MA, Borbolla RS. Prevalencia de los factores de riesgo cardiovascular en trabajadores de una planta metalúrgica. Med. segur. trab. 2012; 58 (228): 269-281.

16. Zimmermann VM, González GM, Galán LI. Perfiles de exposición de riesgo cardiovascular según la ocupación laboral en la Comunidad de Madrid. Rev. Esp. Salud Pública. 2010; 84 (3): 293-308.

17. López GA, Angullo ME, Román RM, Vicente HM, Tomás SM, Ricci Cl. Prevalencia de factores de riesgo cardiovascular en trabajadores aparentemente sanos. Gaceta Médica de México. 2012; 148: 430437.

18. Conn VS1, Hafdahl AR, Cooper PS, Brown LM, Lusk SL. Meta-analysis of workplace physical activity interventions. Am J Prev Med. 2009; 37(4):330-339.

19. Mastrangelo G, Marangi G, Bontadi D, Fadda E, Cegolon L, Bortolotto $M$, et al. A worksite intervention to reduce the cardiovascular risk: proposal of a study design easy to integrate within Italian organization of occupational health surveillance. BMC Public Health. 2015; 15 (12): 1-8.

Las opiniones que contenga este artículo son de su(s) autor(es) y no necesariamente
representan la opinión oficial de la Universidad Simón Bolívar, la Revista Ciencia e Innovación en Salud o de sus autoridades. 